\title{
ARGO-YBJ measurements on the EAS-footprint stretching as an effect of the geomagnetic field
}

\author{
P. Bernardini, I. De Mitri*, G. Marsella, L. Perrone (ARGO-YBJ Collaboration) \\ Dipartimento di Matematica e Fisica, Università del Salento \& INFN, sezione di Lecce, Italy \\ E-mail: paolo.bernardini@le.infn.it
}

The ARGO-YBJ experiment proved the effect of the geomagnetic field (GeoMF) on the development of extensive air showers (EAS) and the dependence of the trigger rate on the coupling angle between GeoMF and EAS axis. For showers with the core on the detector the trigger rate decreases with the EAS-GeoMF coupling. When the core is very far and the detector is triggered by the EAS tail the trigger rate is enhanced by the GeoMF effect. As a consequence in both cases the azimuthal distribution is not flat. Here we discuss in detail the GeoMF effect by selecting showers as a function of their distance from the array center and analysing the stretching of the charged particles due to the Lorentz force in the lateral distribution. The expected correlation is fully confirmed.

The 34th International Cosmic Ray Conference,

30 July- 6 August, 2015

The Hague, The Netherlands

*Speaker. 


\section{Introduction}

Cosmic rays (CRs) are charged particles and their paths are deflected by magnetic forces. The geomagnetic field (GeoMF) restrains low-rigidity CRs from reaching the terrestrial atmosphere and causes the well-known East-West effect. The GeoMF acts also on the charged particles of the extensive air showers (EAS) and Cocconi [1] suggested that the lateral displacement induced by the Earth magnetic field is significant at high altitudes. Moreover if the trigger efficiency of a detector is sensitive to the EAS charge density, the trigger rate exhibits a dependence on the angle between GeoMF and EAS axis, therefore a dependence on EAS zenith and azimuth angles. This effect has been observed by the Yakutsk array [2] for showers with the core far away from the detector. ARGO-YBJ experiment [3] confirmed the Yakutsk result and made an unprecedented measurement on the effect of the GeoMF on the development of EAS in proximity of the core. Here we present more details on the trigger modulation due to GeoMF and investigate the magnetic stretching of the shower front.

\section{ARGO-YBJ detector}

The ARGO-YBJ array [4] stopped data-taking in February 2013 (a sketch of the array is visible in Fig. 1) after more than 5 years of operation in the complete set-up. It was located in the YangBaJing Cosmic Ray Observatory (Tibet, P.R. of China) at $4300 \mathrm{~m}$ above sea level $\left(90^{\circ} 31^{\prime} 50^{\prime \prime} E\right.$, $30^{\circ} 06^{\prime} 38^{\prime \prime} N$ ) and was mainly devoted to $\gamma$-astronomy and CR physics. The array was composed of 1560 Resistive Plate Counters (RPCs) arranged in the central full-coverage carpet, plus 276 RPCs in the guard-ring area. The RPC digital read-out was performed by means of 10 external pads (each pad was the logical OR of 8 inductive strips). Each RPC was also equipped with two large pads for analog read-out [5].

ARGO-YBJ operated in two acquisition modes: shower and scaler mode [6]. An electronic logic was applied in shower mode to build an inclusive trigger based on the time correlation between the pad signals, depending on their relative distances. The shower-mode trigger required at least 20 fired pads on the central carpet $\left(74 \times 78 \mathrm{~m}^{2}\right)$ in a time window of $420 \mathrm{~ns}$.

In this analysis the data collected from the digital read-out in shower mode have been considered. So the analysed showers are typically in the energy range $1-200 \mathrm{TeV}$, well beyond the rigidity cutoff at the YBJ site $(\sim 15 G V)$. Therefore the effect of the GeoMF on the primary trajectory is negligible.

\section{Magnetic effect on the trigger rate}

This analysis is essentially devoted to study the dependence of the trigger rate and EAS features on $\xi$, that is the coupling angle between GeoMF and EAS axis. Obviously $\xi$ is a function of $\theta_{B}=46.4^{\circ}, \phi_{B}=71.89^{\circ}$ (zenith and azimuth of the GeoMF in the ARGO-YBJ reference system) and $\theta, \phi$ (zenith and azimuth of the shower). Furthermore it is useful to observe that $\sin \xi$ is always positive for downgoing particles. We have shown [3] that the EAS particles are shifted with respect to the original path because of the Lorentz force. This shift $(d)$ on the shower front is typically 


$$
d=\frac{q L^{2}}{2 p} B \sin \xi
$$

where $q$ is the charge, $L$ the path, $p$ the momentum of the particle and $B$ the GeoMF. This shift reduces the charge density close to the EAS axis. As a consequence also the trigger rate $(\lambda)$ depends on the coupling angle $\xi$ between GeoMF and EAS axis. To be coherent with the previous paper [3] we use the formula

$$
\lambda=\lambda_{0}\left(1-\eta \sin ^{2} \xi\right)
$$

The $\lambda_{0}$ parameter depends on the core position and on the zenith angle. Neglecting some small detector effect the dependence on the azimuth angle is folded in the term $\sin ^{2} \xi$. We verified that $\eta$ is variable, it is positive (negative) for showers with the core inside (outside) the trigger carpet. Therefore the parameter $\lambda_{0}$ represents the largest (smallest) trigger rate for internal (external) showers. A schematic explanation of this result is presented in Fig. 1: the GeoMF reduces the charge density in proximity of the EAS core and enhances it on the EAS tails. The effect on the trigger efficiency is a simple consequence.

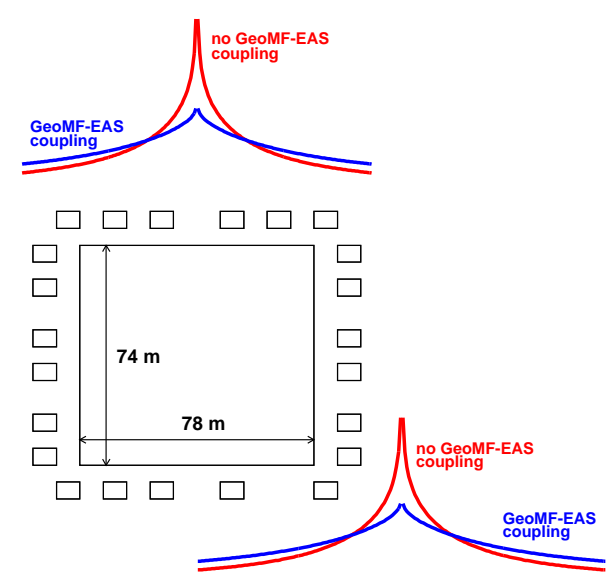

Figure 1: Detector array and sketch of the GeoMF effect on the EAS lateral distribution function (LDF). The EAS core can be on (upper sketch) or outside the trigger carpet (lower sketch). Red: LDF without GeoMF-EAS coupling. Blue: LDF with GeoMF-EAS coupling.

In order to examine in depth the dependence of $\eta$ on the core position and to confirm that the charged-particle lateral distribution is stretched by the magnetic forces, an 8-days data sample has been split in subsamples according to zenith $\left(2^{\circ}\right.$ intervals $)$ and azimuth $\left(5^{\circ}\right.$ intervals $)$ angles and core position. Square belts (10 $\mathrm{m}$ large) have been introduced and their size $\Delta$ is used to describe the core position (see Fig. 2).

In Fig. 3 the zenith angle is fixed, then the $\sin ^{2} \xi$ value ranges with the azimuth angle. Two different belt sizes have been used (the first one is internal, the second one is external to the carpet). The result is very clear: the experimental points are well fitted by means of function (3.2) but 


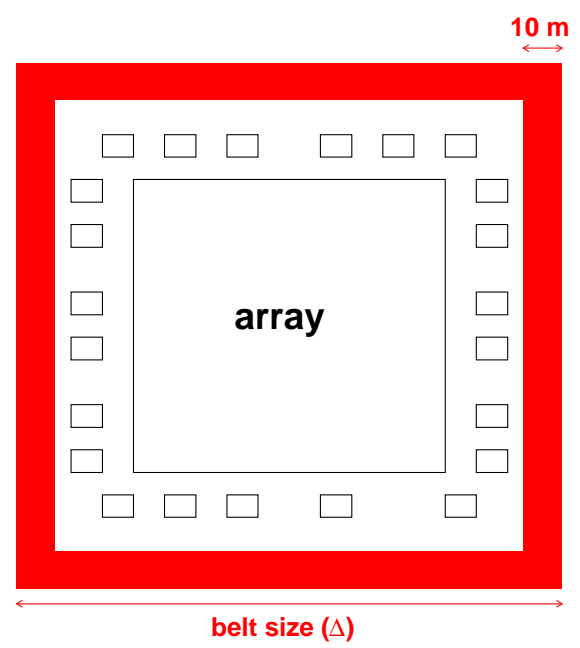

Figure 2: The GeoMF effect has been studied selecting event subsamples according to the size $(\Delta)$ of the belt where the EAS core is. The squared belts have a width of $10 \mathrm{~m}$ and are centered with respect to the array.
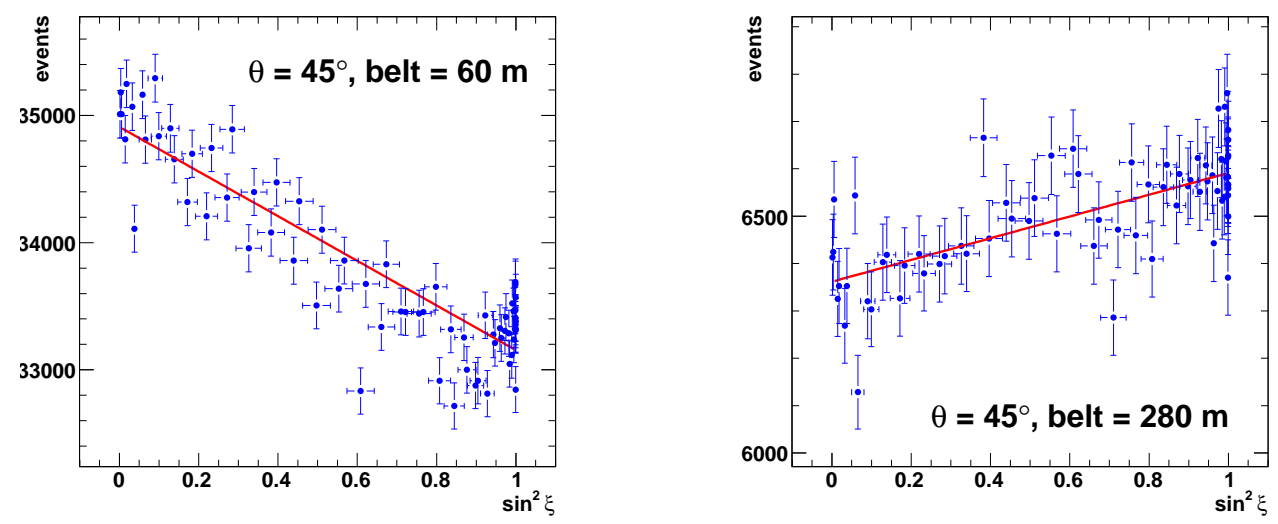

Figure 3: The number of events is plotted as a function of $\sin ^{2} \xi$ for the same zenith range $\left(44^{\circ}<\theta<46^{\circ}\right)$ and different belt sizes $(\Delta)$. For $\Delta=60 \mathrm{~m}$ (left) the rate is reduced by the GeoMF-EAS coupling, for $\Delta=280 m$ (right) the rate is increased.

$\eta$ is positive for the internal belt (decreasing rate with $\sin ^{2} \xi$ ) and negative for the external belt (increasing rate with $\sin ^{2} \xi$ ). This approach has been repeated for all $\theta$-values and belt sizes up to $500 \mathrm{~m}$. The $\eta$ values have been estimated by means of fit and the profile of $\eta$ versus the belt size is shown in Fig. 4. Different regimes are visible. The $\eta$ value is positive in the range $0-170 \mathrm{~m}$ ( $\mathrm{A}+\mathrm{B}$ ranges) and negative for $\Delta>170 m(\mathrm{C}+\mathrm{D}$ ranges). Therefore in the $\mathrm{A}$ and $\mathrm{B}$ ranges the GeoMF reduces the number of charged particles useful for the trigger. The highest $\eta$ value (almost $7 \%)$ is reached on the boundaries of the detector $(\Delta \sim 90 \mathrm{~m})$. When the core is far away from the detector $(\Delta>170 \mathrm{~m})$ the GeoMF increases the charge density on the tails of the showers, therefore 
the trigger rate increases with $\sin ^{2} \xi$ and $\eta$ is negative. In the range $\mathrm{D}(\Delta>300 \mathrm{~m})$ the GeoMF effect becomes stable $(\eta \simeq-4.4 \%)$. When the core is that far the shower reconstruction is worse and the shower front is very large in time. In these conditions the increase of the GeoMF effect is overwhelmed.

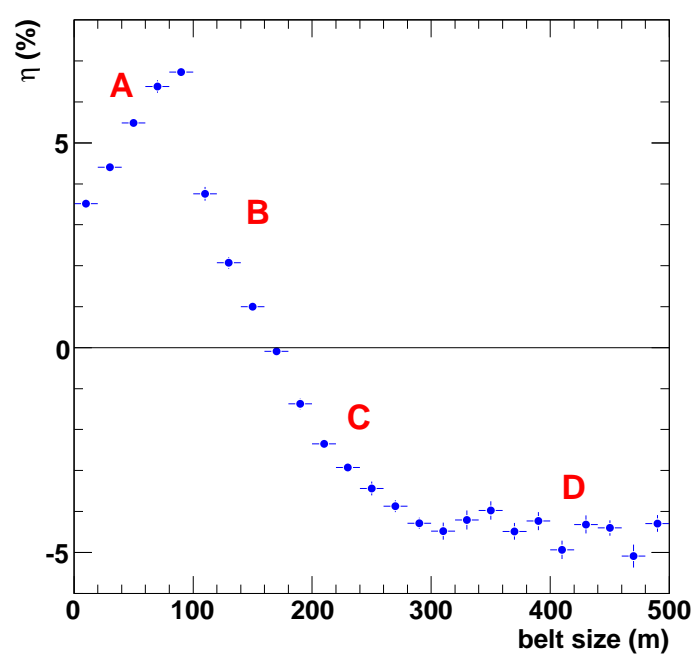

Figure 4: Profile of the $\eta$ coefficient as a function of the size of the belt where the EAS core is.

The simple model shown in Fig. 1 and presented in detail in [3] is confirmed. The GeoMF moves charged particles off from the EAS axis and enhances the charge density on the tails. To show the effect of the different regimes $(\eta>0$ and $\eta<0)$ the azimuthal distribution is shown in Fig. 5 for two different belt ranges. As expected the difference is very strong, indeed both distributions can be fitted with a double harmonic function but the coefficients have opposite sign. More details about the analysis of the azimuthal distribution are available in [3].
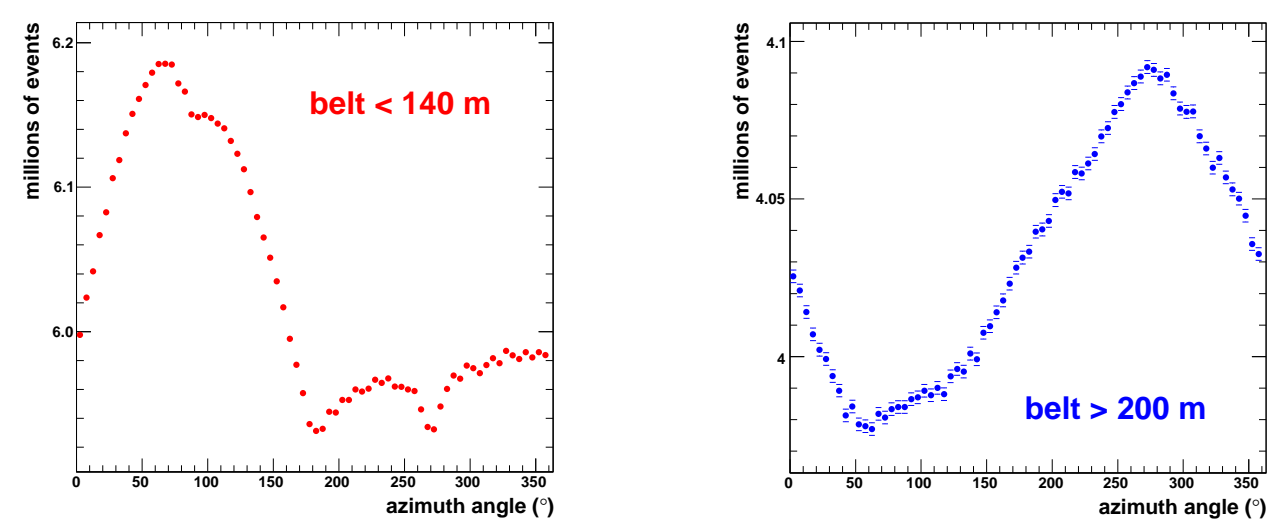

Figure 5: Azimuthal distribution for two subsamples. Left: the cut $\Delta<140 m$ involves the condition $\eta>0$ (rate decrease with the EAS-GeoMF coupling). Right: the cut $\Delta>200 m$ means $\eta<0$ (rate increase with the EAS-GeoMF coupling). 

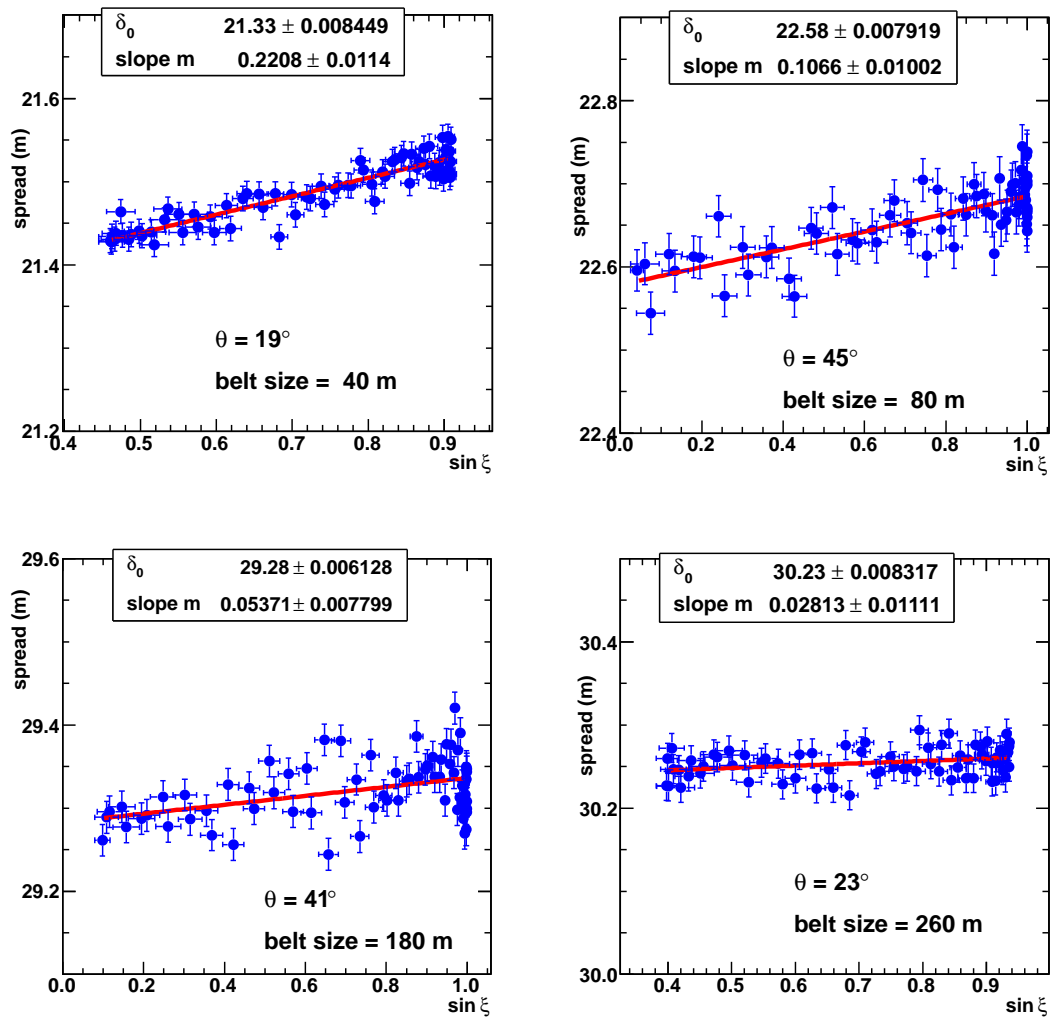

Figure 6: Examples of the spread dependence on the EAS-GeoMF coupling. The spread is always increasing with $\sin \xi(m>0)$.

\section{Magnetic effect on the particle density}

Based on the arguments discussed in the previous section, we conclude that the mutual distances among hits are increased because of the GeoMF. As a consequence, the analysis of the distance of each hit from the hit barycenter could provide a check of the discussed results. Therefore we introduce the spread $\delta$, that is the mean hit-barycenter distance, defined as

$$
\delta=\frac{\sum \delta_{i} w_{i}}{\sum w_{i}}
$$

Here the sum is over all the fired pads in the event, $\delta_{i}$ is the pad-barycenter distance and $w_{i}$ is the number of strips fired on the $i$-th pad. It is expected that the GeoMF shift of positive and negative charges is the same $(d)$ but in reverse directions. According to eq. (3.1) and assuming that the barycenter is fixed, that is the number of positive shower particles is about the same of negative ones, $\delta$ increases with $\sin \xi$ following the formula

$$
\delta=\delta_{0}+\langle d \Gamma\rangle=\delta_{0}+\left\langle\frac{q L^{2} B}{2 p} \Gamma\right\rangle \sin \xi=\delta_{0}+m \sin \xi,
$$

where $\delta_{0}$ is the spread without GeoMF and the $\Gamma$ term represents the correction necessary to take into account that $d$ is on the shower front and $\delta$ is on the detector plane. The linearity of $\delta$ vs $\sin \xi$ is 
confirmed from the data (some examples for fixed zenith and belt-size values are shown in Fig. 6). This check has been repeated for $\theta$ in the range $6^{\circ}-60^{\circ}$ and $\Delta<500 \mathrm{~m}$. Lower $\theta$-values do not allow a reliable fit because the $\sin \xi$-range is too short $\left(0.65<\sin \xi<0.8\right.$ at $\left.\theta=6^{\circ}\right)$. Furthermore the shower reconstruction is less accurate for high zenith angle and very far core. Indeed the cut $\theta<60^{\circ}$ has been used also in [3].

The result of this analysis is in agreement with the model: the spread is always increasing with $\sin \xi$, for internal and external cores. The increase of the spread on the carpet with respect to $\sin \xi$ (the slope coefficient $m$ ) is shown in Fig. 7 as a function of the belt size. We observe that the slope is higher when the core is on the carpet and then falls down to a minimum value. The measurement of $m$ is an estimate of the shift of the particles on the detector plane due to the GeoMF. Indeed the slope in Fig. 7 is the $\delta$ variation on the detector plane for $\sin \xi$ ranging from 0 to 1 . The highest shift is $\sim 20 \mathrm{~cm}$ for showers with the core at the center of the carpet. The lowest value is $\sim 3 \mathrm{~cm}$ for showers with the external core.

For sake of completeness it should be noted that the full range (0-1) of $\sin \xi$ is accessible only for $\theta \simeq 45^{\circ}$. For the other zenith angles the range of $\sin \xi$ is shorter (see the previous example for $\theta=6^{\circ}$ ) Then the $\delta$ variation due to the GeoMF is limited. Furthermore the increase must be compared with spreads of $20-30 \mathrm{~m}$, that is the increase is much much lower than $1 \%$, also when $\sin \xi$ ranges from 0 to 1 .

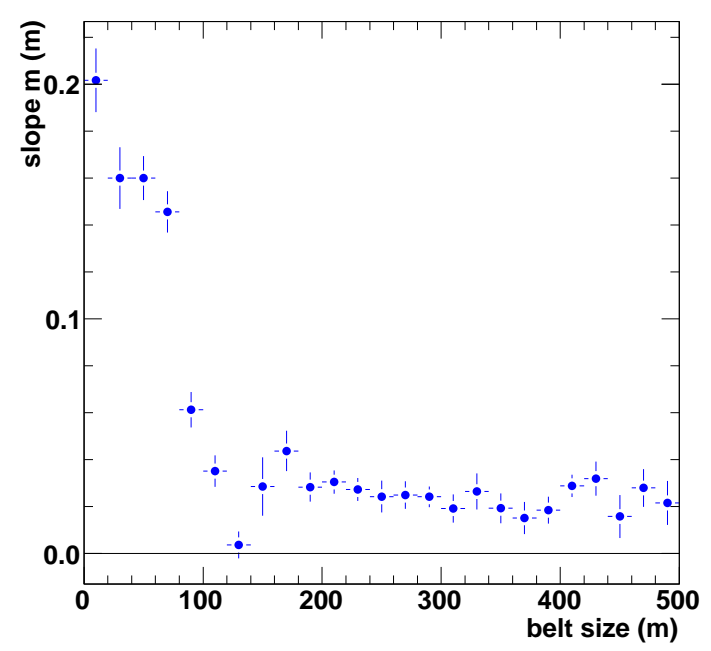

Figure 7: The increase of the spread on the carpet with respect to $\sin \xi$ (slope $m$ ) is plotted as a function of the belt size. The slope- $m$ value is strongly reduced for EAS cores external to the carpet (belt size $>80 \mathrm{~m}$ ).

\section{Conclusions}

The data collected by the ARGO-YBJ detector have been analysed looking for the GeoMF effect on the EAS development. The dependence of the trigger rate on the EAS-GeoMF coupling and the core distance from the detector center has been confirmed. Despite the GeoMF effect is very small, the analysis of the shower spread allowed to verify and to measure the typical shift of 
the charged particles on the detector plane due to the Lorentz force. The high granularity and the full coverage of the ARGO-YBJ detector allowed to observe also a shift of few $\mathrm{cm}$. Finally we want to stress that the fit quality confirms that the shift $d$ depends on $\sin \xi$ (eq. (3.1)) whereas the rate $\lambda$ depends on $\sin ^{2} \xi$ (eq. (3.2)).

\section{References}

[1] G. Cocconi, Phys. Review 93 (1954) 646; erratum, Phys. Review 95 (1954) 1705

[2] A.A. Ivanov et al., JETP Letters 69 (1999) 288

[3] B. Bartoli et al. (ARGO-YBJ Collaboration), Phys. Rev. D 89 (2014) 052005

[4] G. Aielli et al. (ARGO-YBJ Collaboration), Nuclear Instrum. Methods A 661 (2012) S50

[5] B. Bartoli et al. (ARGO-YBJ Collaboration), Astropart. Physics 67 (2015) 47

[6] G. Aielli et al. (ARGO-YBJ Collaboration), Astropart. Physics 30 (2008) 85; G. Aielli et al. (ARGO-YBJ Collaboration), Astroph. Journal 699 (2009) 1281 